\title{
Association of serum cystatin C levels with mortality in patients with acute type $\mathrm{A}$ aortic dissection
}

\author{
Wei-Zhong Feng ${ }^{1}$, Jun-Qing Zhou ${ }^{1}$, Guang-Mao $\mathbf{Y u}^{1}$, Yong Zeng ${ }^{1}$ and Peng $\mathrm{Xu}^{1}$ \\ ${ }^{1}$ Department of Cardiothoracic Surgery, Shaoxing People's Hospital, Shaoxing Hospital of Zhejiang University, Shaoxing \\ 312000, Zhejiang Province, China
}

Correspondence to: Jun-Qing Zhou, email: jun_qingzhou@sina.com

Guang-Mao Yu, email: guangmaoyu0@outlook.com

Keywords: acute type A aortic dissection, cystatin C, high sensitive C-reactive protein, mortality, prognosis

Received: June 15, $2017 \quad$ Accepted: July 26, $2017 \quad$ Published: August 30, 2017

Copyright: Feng et al. This is an open-access article distributed under the terms of the Creative Commons Attribution License 3.0 (CC BY $3.0)$, which permits unrestricted use, distribution, and reproduction in any medium, provided the original author and source are credited.

\section{ABSTRACT}

Increased serum cystatin C levels are related to the prognosis of cardiovascular diseases. This study aims to investigate the effect of admission serum cystatin $C$ levels on short- and long-term mortality in patients with acute type A aortic dissection (ATAAD). From 2010 to 2014, 136 consecutive patients with ATAAD were enrolled and followed up. Clinical data and laboratory assays including were measured. During

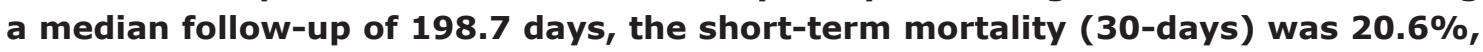
whereas the long-term death rate was $10.2 \%$. We identified that the expression of cystatin C and high-sensitivity C-reactive protein (hs-CRP) in the dying patients was higher than in the surviving patients $(P<0.01)$. Hs-CRP $(H R=1.41,95 \%$ CI: 1.03-2.59, $P=0.037$ ) was an independent risk factor of short-term death determined by univariate and multivariate Cox analyses. No impact of cystatin $\mathrm{C}$ was observed on the short-term mortality. For long-term mortality, cystatin C (HR $=1.49,95 \%$ CI: 1.10-7.36, $P=0.013$ ) was identified as an independent predictor at above the cutoff value $\geq 1.10 \mathrm{mg} / \mathrm{L}$. ROC analysis showed the AUC values of cystatin C and hs-CRP were 0.772 (95\% CI, 0.692-0.839) and 0.640 (95\% CI, 0.574-0.739), respectively, in the prediction of long-term death. The combined AUC value of cystatin C and hsCRP was 0.883 (95\% CI, 0.826-0.935; P < 0.01). Taken together, high cystatin C levels ( $\geq 1.10 \mathrm{mg} / \mathrm{L}$ ) on admission are independently associated with the long-term mortality in patients with ATAAD.

\section{INTRODUCTION}

Acute aortic dissection (AAD) is a devastating cardiovascular condition with an incidence of about 2.6-3.6 cases per 100,000 people per year [1]. AAD is characterized by rapid development of an intimal flap, which is caused by blood flow into the media that divides the intima and the adventitia. This intimal flap separates the true lumen from a false lumen [2]. Acute type-A aortic dissection (ATAAD), when the ascending aortic thoracic tract is involved, occurs in almost $75 \%$ of patients with AAD. This can lead to a mortality rate reaching $90 \%$ if untreated and usually requires swift open surgical repair [3]. Thus, identification of the predictors for short- or longterm mortality of patients with ATAAD may contribute to reduced mortality. Concentrations of white blood cell count (WBCc) [4], C-reactive protein (CRP) [5], D-dimer [6], cardiac troponin $\mathrm{T}$ [7] and matrix metalloproteinases [8] have shown to be associated with the prognosis of AAD.

Cystatin $\mathrm{C}$ is a type of cysteine protease inhibitor whose levels increase much earlier than those of urea and creatinine when renal function declines $[9,10]$. Previous studies have also illustrated an important role of cystatin $\mathrm{C}$ in arterial remodeling and atherogenesis [11]. More importantly, many studies have shown that an elevated serum cystatin $\mathrm{C}$ level was independently associated with an increased risk of cardiovascular or all-cause mortality $[12,13]$, and this predictive function may not only result from a marker for detection of renal impairment [14]. To 
date, no study has determined the prognostic significance of cystatin $\mathrm{C}$ in patients with ATAAD. Therefore, this study was designed to determine the diagnostic value of cystatin $\mathrm{C}$ in predicting the short- and long-term prognosis of patients with ATAAD.

\section{RESULTS}

\section{Baseline patient demographics}

A total of 136 patients were enrolled in this study, including 77 males and 59 females, with an average age of $53.7 \pm 10.3$ years. Patient demographics and baseline characteristics are shown in Table 1. After the follow-up period, 97 patients survived and 39 died ( 28 patients died during short-term follow up and 11 during long-term). The causes of death including aortic rupture (20), myocardial infarction (4), heart failure (6), stroke (4) and valvular dysfunction (5). In our findings, the ascending aorta diameter was larger in the death group when compared to the survival group $(P=0.034)$. Patients who received surgical treatment were more likely to survive $(P<0.001)$. No significant differences in patients' age, sex, body mass index, and cardiovascular risks such as hypertension and diabetes mellitus were observed between the death and survival patient groups.

With respect to laboratory findings, WBCc $(14.7 \pm 4.8$ $\times 10^{9}$ vs $12.8 \pm 4.0 \times 10^{9}$ cells $\left./ \mathrm{L}, P=0.019\right)$, hs-CRP $(5.8 \pm$ 2.1 vs $4.2 \pm 2.3 \mathrm{mg} / \mathrm{L}, P<0.001)$, D-dimer ( $7.6 \pm 3.5$ vs 5.9 $\pm 3.0 \mu \mathrm{g} / \mathrm{mL}, P=0.005)$, high-sensitivity troponin I (hs-cTnI) $(0.029 \pm 0.021$ vs $0.011 \pm 0.010 \mathrm{ng} / \mathrm{mL}, P<0.001)$, BNP $(584 \pm 415$ vs $430 \pm 402 \mathrm{pg} / \mathrm{ml}, P=0.047)$, and cystatin C $(1.20 \pm 0.5$ vs $0.89 \pm 0.3 \mathrm{mg} / \mathrm{L}, P<0.001)$ concentrations were significantly higher in the death group than in the survival group. The comparison of cystatin $\mathrm{C}$ and hs-CRP levels between the two groups are shown in Figure 1.

\section{Correlation of cystatin $C$ with hs-CRP in ATAAD}

As shown in Figure 2, serum cystatin $\mathrm{C}$ levels existed positive correlation to hs-CRP concentrations, $r=0.4675(P<0.001)$, which were analyzed by the spearman correlation; serum cystatin $\mathrm{C}$ was also correlated with WBCc $(r=0.2305, P=0.001)$, but not D-dimer ( $r=0.1067, P=0.134$; not shown).

\section{Analysis of risk factors for short- and long-term mortality}

As shown in Figure 3A, Kaplan-Meier survival curves stratified by admission serum cystatin $\mathrm{C}$ levels showed no difference in short-term survival detected by the $\log$ rank test $(P=0.3917)$. Interestingly, the log rank test identified a significant difference in long-term survival among the patients stratified by serum cystatin $\mathrm{C}$ (Figure 3B, $P=0.006$ ).

Univariate and multivariate Cox analyses for short- and long-term mortality in the enrolled patients are presented in Table 2 and Table 3, respectively. The data revealed that seven variables were associated with shortterm mortality $(20.6 \%, 28 / 136)$ including ascending aorta diameter, surgical treatment, WBCc, hs-CRP, D-dimer, hs-cTnI, and cystatin C. After multivariable adjustment, hs-CRP (HR $=1.41,95 \%$ CI: $1.03-2.59, P=0.037)$ was an independent risk factor, whereas surgical treatment (HR $=0.12,95 \%$ CI:0.01-0.41, $P<0.001)$ was an independent protective factor for short-term mortality (Table 2). During the long-term follow-up, the all-cause mortality was $10.2 \%(11 / 108)$. Univariate analysis revealed three associated variables and multivariable analysis indicated that serum cystatin $\mathrm{C}(\mathrm{HR}=1.49,95 \% \mathrm{CI}: 1.10-7.36$, $P=0.013)$ and surgical treatment $(\mathrm{HR}=0.22,95 \%$ CI:0.03-0.57, $P<0.001)$ were independent predictors of long-term mortality (Table 3 ).
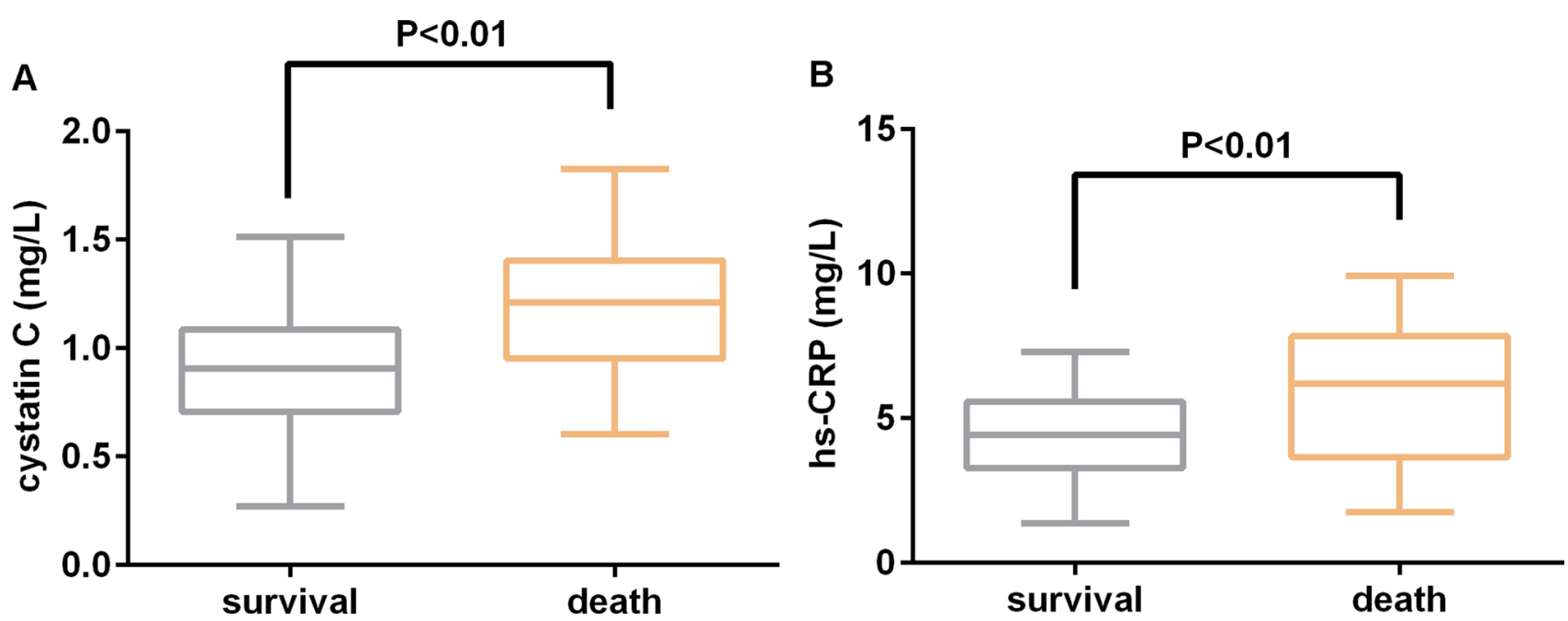

Figure 1: Comparison of (A) serum cystatin $C$ and high-sensitivity C-reactive protein (hs-CRP) (B) values between survival and death patients admitted with acute Type A aortic dissection. 
Table 1: Baseline characteristics of patients with type A acute aortic dissection (AAD)

\begin{tabular}{|c|c|c|c|c|}
\hline Clinical variables & Survival $(n=97)$ & $\operatorname{Death}(n=39)$ & Statistics & $P$-value \\
\hline Age(years) & $52.8 \pm 9.6$ & $54.6 \pm 11.5$ & $0.933^{\mathrm{a}}$ & 0.352 \\
\hline Male Sex $n(\%)$ & $57(58.8 \%)$ & $20(51.3 \%)$ & $0.192^{b}$ & 0.660 \\
\hline Body mass index $(\mathrm{kg} / \mathrm{m} 2)$ & $24.7 \pm 3.6$ & $25.4 \pm 2.8$ & $1.088^{\mathrm{a}}$ & 0.278 \\
\hline History of smoking & $31(31.9 \%)$ & $18(46.2 \%)$ & $2.678^{b}$ & 0.101 \\
\hline Hypertension & $65(67.0 \%)$ & $31(79.5 \%)$ & $1.528^{\mathrm{b}}$ & 0.216 \\
\hline Diabetes mellitus & $19(19.6 \%)$ & $12(30.8 \%)$ & $1.392^{\mathrm{b}}$ & 0.238 \\
\hline Admission SBP (mmHg) & $164.5 \pm 25.3$ & $170.2 \pm 26.8$ & $1.168^{\mathrm{a}}$ & 0.242 \\
\hline Admission SBP (mmHg) & $79.2 \pm 14.6$ & $82.8 \pm 19.2$ & $1.184^{\mathrm{a}}$ & 0.238 \\
\hline $\mathrm{AAD}, \mathrm{mm}$ & $44.6 \pm 6.1$ & $47.0 \pm 5.5$ & $2.132^{\mathrm{a}}$ & 0.034 \\
\hline Aspirin & $16(16.5 \%)$ & $6(15.4 \%)$ & $0.009^{\mathrm{b}}$ & 0.922 \\
\hline Nitroglycerin & $38(39.2 \%)$ & $11(28.2 \%)$ & $1.015^{\mathrm{b}}$ & 0.314 \\
\hline Surgical treatment, $n(\%)$ & $79(81.4 \%)$ & $20(51.3 \%)$ & $11.30^{\mathrm{b}}$ & $<0.001$ \\
\hline Total cholesterol $(\mathrm{mmol} / \mathrm{L})$ & $165.3 \pm 24.8$ & $170.0 \pm 30.3$ & $0.936^{\mathrm{a}}$ & 0.351 \\
\hline LDL-C (mg/dL ) & $95.3 \pm 18.7$ & $97.2 \pm 23.1$ & $0.499^{\mathrm{a}}$ & 0.618 \\
\hline WBCc $\left(\times 10^{9}\right.$ cells $\left./ \mathrm{L}\right)$ & $12.8 \pm 4.0$ & $14.7 \pm 4.8$ & $2.362^{\mathrm{a}}$ & 0.019 \\
\hline hs-CRP (mg/L) & $4.2 \pm 2.3$ & $5.8 \pm 2.1$ & $3.285^{\mathrm{a}}$ & $<0.001$ \\
\hline $\mathrm{D}$-dimer $(\mu \mathrm{g} / \mathrm{mL})$ & $5.9 \pm 3.0$ & $7.6 \pm 3.5$ & $2.864^{\mathrm{a}}$ & 0.005 \\
\hline hs-cTnI (ng/mL) & $0.011 \pm 0.010$ & $0.029 \pm 0.021$ & $6.769^{\mathrm{a}}$ & $<0.001$ \\
\hline $\mathrm{BNP}(\mathrm{pg} / \mathrm{ml})$ & $430 \pm 402$ & $584 \pm 415$ & $2.002^{\mathrm{a}}$ & 0.047 \\
\hline Serum Creatinine $(\mu \mathrm{mol} / \mathrm{L})$ & $93.1 \pm 14.5$ & $95.5 \pm 18.9$ & $0.797^{\mathrm{a}}$ & 0.426 \\
\hline cystatin $\mathrm{C}(\mathrm{mg} / \mathrm{L})$ & $0.89 \pm 0.3$ & $1.20 \pm 0.5$ & $4.444^{\mathrm{a}}$ & $<0.001$ \\
\hline
\end{tabular}

Note: Data are expressed as mean \pm SD. $\mathrm{a}=t$ value; $\mathrm{b}=\chi^{2}$ value; SBP: systolic blood pressure; DBP: diastolic blood pressure; AAD: ascending aorta diameter; LDL-C: low density lipoprotein- cholesterol; WBCc: white blood cell count; hscTnI: high-sensitivity troponin I; BNP: Brain natriuretic peptide; hs-CRP: high sensitive C-reactive protein.

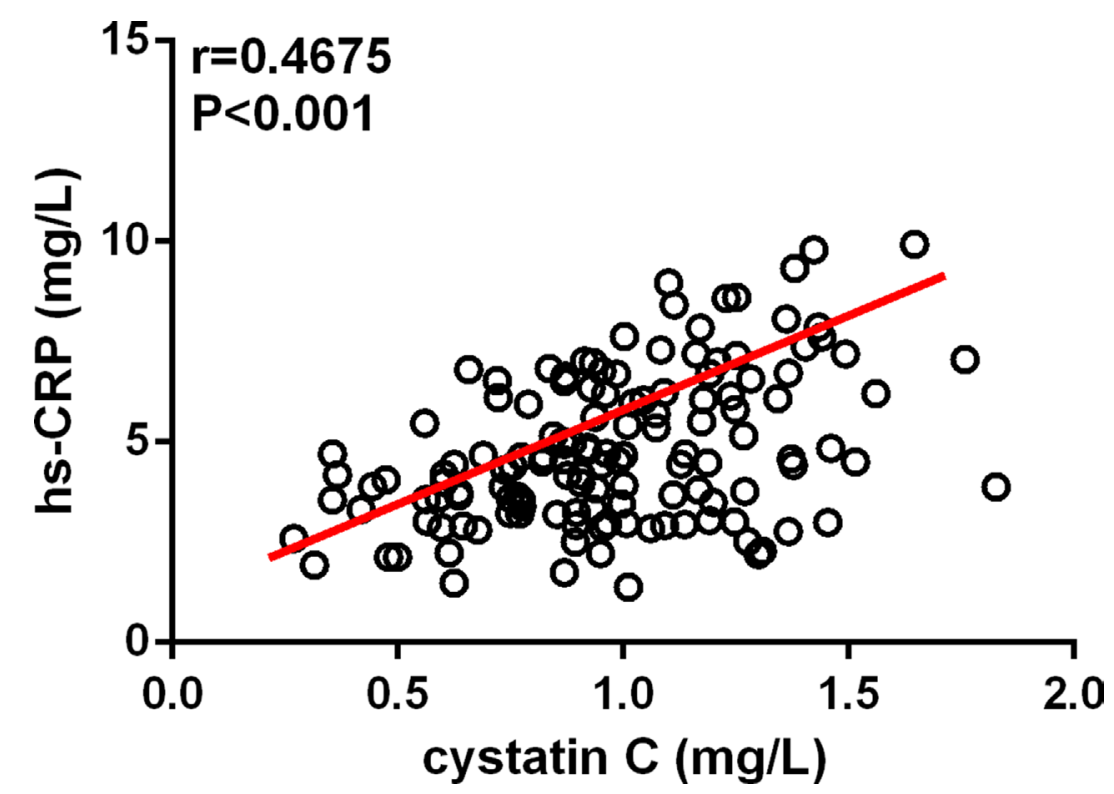

Figure 2: Cystatin C levels showed a positive correlation with high-sensitivity C-reactive protein (hs-CRP) levels in patients with acute type A aortic dissection detected by the Spearman correlation. 
Table 2: Effects of various variables on short-term mortality in univariate and multivariate logistic regression analyses

\begin{tabular}{lcccc}
\hline Variable & \multicolumn{2}{c}{ Univariable } & \multicolumn{2}{c}{ Multivariable } \\
\cline { 2 - 4 } & OR (95\% CI) & $P$-value & OR (95\% CI) & $P$-value \\
\hline Age $>$ 65 years & $1.21(0.82-1.89)$ & 0.312 & & \\
History of smoking & $1.66(0.65-4.67)$ & 0.553 & & \\
Admission SBP $(>140 \mathrm{mmHg})$ & $1.31(0.95-2.09)$ & 0.350 & & \\
Admission DBP $(>90 \mathrm{mmHg})$ & $0.86(0.31-1.58)$ & 0.441 & & \\
AAD, mm & $1.34(0.99-3.31)$ & 0.042 & $1.22(0.97-1.86)$ & 0.134 \\
Surgical treatment & $0.07(0.01-0.35)$ & $<0.001$ & $0.12(0.01-0.41)$ & $<0.001$ \\
WBCc $\left(\times 10^{9}\right.$ cells/L) & $1.98(0.99-4.58)$ & 0.004 & $1.19(0.91-3.16)$ & 0.073 \\
hs-CRP $(\mathrm{mg} / \mathrm{L})$ & $2.11(1.72-8.35)$ & 0.012 & $1.41(1.03-2.59)$ & 0.037 \\
D-dimer $(\mu \mathrm{g} / \mathrm{mL})$ & $4.28(2.10-12.92)$ & $<0.001$ & $3.35(0.82-9.41)$ & 0.109 \\
hs-cTnI $(\mathrm{ng} / \mathrm{mL})$ & $1.65(1.05-1.50)$ & 0.017 & $1.20(0.96-1.33)$ & 0.215 \\
BNP $(\mathrm{pg} / \mathrm{ml})$ & $1.04(0.85-2.90)$ & 0.185 & & \\
Serum Creatinine $(\mu \mathrm{mol} / \mathrm{L})$ & $1.79(0.77-4.46)$ & 0.259 & & 0.238 \\
cystatin C $(\mathrm{mg} / \mathrm{L})$ & $2.54(1.14-7.86)$ & 0.002 & $1.67(0.84-2.87)$ & \\
\hline
\end{tabular}

Note: SBP: systolic blood pressure; DBP: diastolic blood pressure; AAD: acute aortic dissection; WBCc: white blood cell count; hs-cTnI: high-sensitivity troponin I; BNP: Brain natriuretic peptide; hs-CRP: high sensitive C-reactive protein. OR: Odds ratio; CI: Confidence interval.

\section{Diagnostic value of cystatin $\mathrm{C}$ in predicting mortality}

Receiver operating characteristic (ROC) curves were used to further investigate the predictive power of cystatin $\mathrm{C}$ in evaluating long-term mortality. The results indicated that the area under the curve (AUC) value of cystatin $\mathrm{C}$ was 0.772 (95\% CI, 0.692-0.839; Figure 4A), while the AUC of hs-CRP was 0.640 (95\% CI, 0.574-0.739; Figure 4B). When cystatin $\mathrm{C}$ was $\geq 1.10 \mathrm{mg} / \mathrm{L}$, the sensitivity and specificity in predicting long-term death were $78.53 \%$ and $68.23 \%$, respectively. When hs-CRP was $\geq 5.76 \mathrm{mg} / \mathrm{L}$, the sensitivity

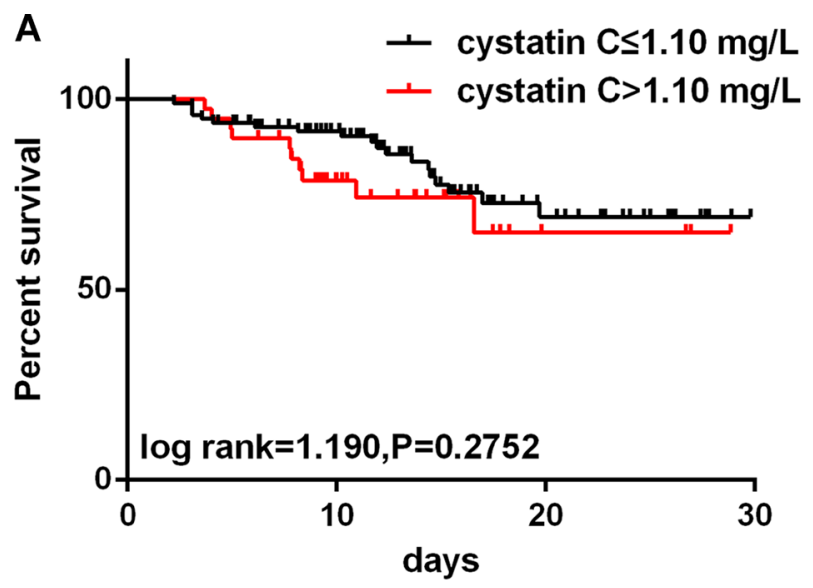

and specificity were $86.72 \%$ and $46.51 \%$, respectively. Furthermore, the ROC curve comparison revealed a higher prognostic value of cystatin $\mathrm{C}+\mathrm{hs}-\mathrm{CRP}$ compared with cystatin $\mathrm{C}$ or hs-CRP alone $(P<0.01)$ as shown in Figure 4C. Cystatin C + hs-CRP yielded the highest AUC values at 0.883 ( $95 \% \mathrm{CI}, 0.826-0.935)$, with a sensitivity of $97.44 \%$ and a specificity of $65.92 \%$ (Figure $4 \mathrm{C}$ ).

\section{DISCUSSION}

This study identified that serum cystatin $\mathrm{C}$ levels in patients with ATAAD had a good correlation with hs-CRP

Figure 3: Kaplan-Meier analyses for short- and long-term mortality according to admission cystatin C (cut-off: 1.10 $\mathbf{m g} / \mathbf{L}$ ) values. (A) Short-term survival curves according to the cystatin C cut-off values $(1.10 \mathrm{mg} / \mathrm{L}$ ). (B) Long-term survival curves according to the cystatin $\mathrm{C}$ cut-off values $(1.10 \mathrm{mg} / \mathrm{L})$. 
Table 3: Effects of various variables on long-term mortality in univariate and multivariate logistic regression analyses

\begin{tabular}{lcccc}
\hline Variable & \multicolumn{2}{c}{ Univariable } & Multivariable \\
\cline { 2 - 4 } & OR (95\% CI) & $\boldsymbol{P}$-value & OR (95\% CI) & $\boldsymbol{P}$-value \\
\hline Age $>$ 65 years & $1.51(0.95-3.88)$ & 0.032 & $1.20(0.83-2.20)$ & 0.406 \\
History of smoking & $1.44(0.71-4.59)$ & 0.318 & & \\
Admission SBP $(>140 \mathrm{mmHg})$ & $0.98(0.42-3.76)$ & 0.417 & & $<0.001$ \\
Admission DBP $(>90 \mathrm{mmHg})$ & $0.74(0.25-2.52)$ & 0.671 & & \\
AAD, mm & $1.14(0.93-2.29)$ & 0.172 & & \\
Surgical treatment & $0.15(0.03-0.41)$ & $<0.001$ & $0.22(0.03-0.57)$ & \\
WBCc $\left(\times 10^{9}\right.$ cells/L) & $1.38(0.74-6.42)$ & 0.211 & & \\
hs-CRP $(\mathrm{mg} / \mathrm{L})$ & $1.21(0.82-5.49)$ & 0.134 & & \\
D-dimer $(\mu \mathrm{g} / \mathrm{mL})$ & $1.98(0.86-4.67)$ & 0.320 & & \\
hs-cTnI $(\mathrm{ng} / \mathrm{mL})$ & $1.10(0.91-1.21)$ & 0.147 & & \\
BNP $(\mathrm{pg} / \mathrm{ml})$ & $1.02(0.78-1.06)$ & 0.343 & & \\
Serum Creatinine $(\mu \mathrm{mol} / \mathrm{L})$ & $0.93(0.47-2.44)$ & 0.604 & & \\
cystatin C $(\mathrm{mg} / \mathrm{L})$ & $1.94(1.25-9.03)$ & $<0.001$ & $1.49(1.10-7.36)$ & \\
\hline Ne: SBP: sysic blod & & & \\
\hline
\end{tabular}

Note: SBP: systolic blood pressure; DBP: diastolic blood pressure; AAD: acute aortic dissection; WBCc: white blood cell count; hs-cTnI: high-sensitivity troponin I; BNP: Brain natriuretic peptide; hs-CRP: high sensitive C-reactive protein. OR: Odds ratio; CI: Confidence interval.

levels. Although no association was found between serum cystatin $\mathrm{C}$ and short-term mortality, our data showed when using the cut-off value $\geq 1.10 \mathrm{mg} / \mathrm{L}$, cystatin $\mathrm{C}$ was an independent risk factor for long-term prognosis. The sensitivity and specificity in predicting long-term mortality were $78.53 \%$ and 68.23 , respectively, suggesting that cystatin $\mathrm{C}$ on admission is an independent predictor of long-term mortality in patients with ATAAD.
AAD is the main cause of cardiovascular morbidity and mortality. Although no epidemiological data have been confirmed, the number of patients with AAD has increased in China in recent years [15]. ATAAD owns high in-hospital mortality due to potentially fatal complications, such as coronary involvement, even in centers that have extensive expertise and interest in the treatment of highrisk patients [16]. The reoccurrence of aortic dissection in
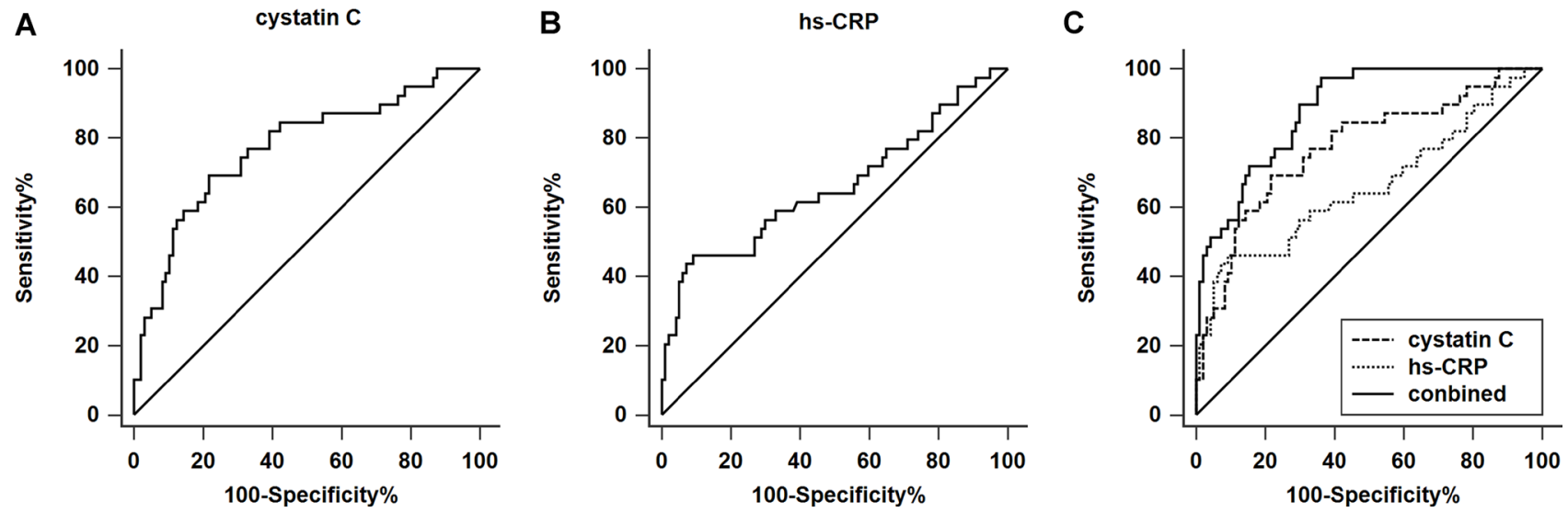

Figure 4: ROC analysis of serum cystatin C and high-sensitivity C-reactive protein (hs-CRP) in the long-term mortality of patients with acute type A aortic dissection. (A) In the ROC analysis of serum cystatin C, the AUC value is 0.772 (95\% CI: $0.692-0.839$ ) with a sensitivity and specificity of $78.53 \%$ and $69.23 \%$, respectively. The cut-off value is $1.10 \mathrm{mg} / \mathrm{L}$. (B) In the ROC analysis of hs-CRP, the AUC value is 0.640 (95\% CI: $0.574-0.739)$ with a sensitivity and specificity of $86.72 \%$ and $46.51 \%$, respectively. The cut-off value is $5.57 \mathrm{mg} / \mathrm{L}$. (C) The AUC value of combined (cystatin C+ hs-CRP) is 0.883 (95\% CI: $0.826-0.935)$ with a sensitivity and specificity of $97.44 \%$ and $65.92 \%$, respectively. The cut-off value is -1.51 . (combined $=-8.40+0.62 *$ hs-CRP $+0.45 *$ cystatin $\mathrm{C}$ ). 
the chronic phase may also contribute to the high mortality [17]. Therefore, classifying high-risk patients with a poor long-term prognosis is clinically important.

Cystatin $\mathrm{C}$ is a non-glycosylated basic protein produced at a constant rate by investigated nucleated cells. It is considered as a novel marker for renal dysfunction, which is better than serum creatinine, especially for mild renal impairment [18, 19]. Renal impairment is clearly an independent risk factor for cardiovascular disease and a close relationship has been established between cystatin $\mathrm{C}$ and various subsets of cardiovascular diseases, including coronary heart disease [20], acute coronary syndrome [21] and heart failure [10]. Pascual et al. suggested that cystatin $\mathrm{C}$ independently predicts death or heart failure rehospitalization with greater accuracy than creatinine [22]. Various studies found that cystatin $\mathrm{C}$ is also an important prognostic factor of a poor outcome in patients with acute coronary syndrome [23, 24]. These all demonstrated the prognostic significance of cystatin $\mathrm{C}$ in cardiovascular disease. In this work, admission serum cystatin $\mathrm{C}$ was found to be potentially related to poor long-term outcomes in patients with ATAAD. We showed patients with higher levels of admission cystatin $\mathrm{C}(\geq 1.10 \mathrm{mg} / \mathrm{L})$ the exerted long survival time than lower ones. Because cystatin C plays a role in the balance between cysteine proteases and their inhibitor that favors remodeling of the vascular wall [11], a potential explanation of the association between the serum cystatin $\mathrm{C}$ value and the long-term outcomes in ATAAD is that elevated cystatin $C$ values might affect the remodeling of the dissected aortic wall. Studies by Sukhova et al. [25] and Liu et al. [11] supported our hypothesis. As for short-term mortality, cystatin C showed no sufficient power to predict outcomes when classified using the cut-off value at $1.10 \mathrm{mg} / \mathrm{L}$. A recent study by Mori et al. showed that thoracic endovascular aortic repair of lesions in the descending thoracic aorta has no influence on the level of cystatin $\mathrm{C}$ in AAD patients [26]. The expression levels of cystatin $C$ were immune to AAD therapy in a short time, which may explain our findings that cystatin $\mathrm{C}$ could be used to predict long-term rather than short-term mortality.

In addition, cystatin $\mathrm{C}$ was found to correlate with hs-CRP (markers of inflammation), as well as WBCc. One potential reason for this correlation is that like many other markers of inflammation, its serum concentration may be higher in patients with decreased renal clearance. However, few patients in our cohort experienced severe renal impairment. Another reason may be that cystatin $\mathrm{C}$ is a marker more than for renal function and a marker of inflammation [27]. Although numerous studies reported that the cystatin $\mathrm{C}$ level is produced at a constant rate and not affected by such factors, we found that in patients with ATAAD, serum cystatin C level had a good correlation with hs-CRP levels. This was supported by the finding by Knight et al. that serum cystatin $\mathrm{C}$ appears to be influenced by factors other than renal function alone [28]. Inflammation has been reported to be involved in the pathogenesis of AAD and associated with outcomes $[4,29,30]$. CRP is a well-established inflammatory marker [31]. In line with previous studies, hs-CRP (HR $=1.41,95 \%$ CI: $1.03-2.59$ ) was found to be a predictive marker for short-term morality. Nevertheless, there was no association between long-term all-cause mortality and hs-CRP levels, which may be due to hs-CRP as a marker of the acute phase [30, 32]. Contrarily, Mori et al. found admission CR $P$ values might be a useful marker to predict the long-term outcome in acute aortic dissection and they hypothesized that elevated admission CR $P$ values might also affect the remodeling of the dissected aortic wall [33]. This discrepancy might because they investigate the prognostic value of CRP in both type A and type B patients. Thus, further studies with more patients from multicenter are needed to determine the exact significance of CRP in acute aortic dissection.

It is likely that no single biomarker will be perfectly predictive, but identifying the optimum combinations to develop risk stratification may determine whether relevant therapies affecting these biomarkers have the potential to reduce mortality. We finally found that the combined use of both cystatin $\mathrm{C}$ and hs-CRP levels could increase the sensitivity in assessing long-term mortality up to $97.44 \%$. Therefore, according to our data, it may be reasonable to take both cystatin C and hs-CRP into account as the prognostic markers in patients with ATAAD because of the high sensitivity demonstrated. The data suggesting that if both admission cystatin $\mathrm{C}$ and hs-CRP levels were above the cut-off levels in ATAAD patients, the risk of death was significantly increased.

Some limitations of the present study need to be addressed. First, we did not obtain the cystatin $\mathrm{C}$ profiles at regular intervals during the follow up because of high test costs. As a prognostic marker, it needs more information about dynamic alteration of cystatin C. Second, no patients with end-stage renal disease were included in our cohort, and other studies are needed to evaluate how informative this biomarker will be in individuals with renal dysfunction, as it always occurs in progression of this disease [34]. Furthermore, the cystatin C levels were included as categorical variables, and there still exerts residual confounding. Thus, larger prospective multicenter studies are needed to further confirm our findings.

In conclusion, our study preliminary demonstrated that serum cystatin $\mathrm{C} \geq 1.10 \mathrm{mg} / \mathrm{L}$ and hs-CRP $\geq 5.76$ $\mathrm{mg} / \mathrm{L}$ were important risk factors and independently associated with ATAAD long-term mortality. Furthermore, the combination of serum cystatin $\mathrm{C}$ and hs-CRP is a potentially useful biomarker with high sensitivity in predicting long-term death in patients with ATAAD in clinical settings. 


\section{MATERIALS AND METHODS}

\section{Study population}

We enrolled 136 consecutive patients with ATAAD at Shaoxing Hospital between February 2010 and December 2014. Each patient was diagnosed with ATAAD according to the standard guideline [35] assisting with clinical symptoms, history, ultrasound, computed tomography angiography, ae well as coronary angiography. Acute dissection is defined as within 2 weeks of symptom onset [36]. Exclusion criteria: a) a history of AD episodes, traumatic $\mathrm{AD}$, aortic surgery, and/or endovascular interventions; b) severe organ dysfunction, such as liver or kidney failure; c) Marfan syndrome and those treated with steroids for long-term use; d) incomplete information for an earlier death during the study. The standard data entry form included information on patient demographics, history, complications, imaging study results, details of medical and surgical treatment (including emergency and selective surgery), results of laboratory assays, and clinical outcomes. Patients were followed up every day in-hospital (outcome of death caused by aortic rupture, perioperative death or other causes) and a follow up protocol was conducted after discharge that included clinical assessment and imaging of the aorta (the endpoint was the occurrence of death or loss to follow-up). The short-term mortality was defined as death within 30 days. The mean follow-up time was 198.7 (2-909) days. Hypertension was defined as a systolic blood pressure $>140 \mathrm{~mm} \mathrm{Hg}$ and/or diastolic blood pressure $>90 \mathrm{~mm} \mathrm{Hg}$ or the use of antihypertensive drugs. The definition of diabetes mellitus was that $\mathrm{HbAlc} \geq 6.5 \%$, random plasma glucose $\geq 200 \mathrm{mg} / \mathrm{dl}$, fasting plasma glucose $\geq 126 \mathrm{mg} / \mathrm{dl}$ or OGTT 2-hour glucose in venous plasma $\geq 200 \mathrm{mg} / \mathrm{dl}$, or was receiving hypoglycemic treatments. The study was approved by the ethics committee of the Shaoxing People's Hospital, and written consent was obtained from each subject.

\section{Serum collection and testing}

Venous blood samples were drawn into ethylenediaminetetra-acetic acid (EDTA)-containing tubes from all patients at the first day upon admission before administration of any medication. Supernatants were obtained within $1 \mathrm{~h}$ after centrifugation $\left(4^{\circ} \mathrm{C}, 3500 \mathrm{r} /\right.$ $\mathrm{min}, 10 \mathrm{~min}$ ) and the serum and plasma were immediately stored at $-80^{\circ} \mathrm{C}$ until further analysis. The serum levels of cystatin $\mathrm{C}$ were measured using enzyme-linked immunosorbent assay kits (Yanjin Biological, Shanghai, People's Republic of China) as previously described [37]. Plasma hs-CRP levels were measured via the colorimetric method with a chemistry analyzer (AU5800 Analyzer, Beckman Coulter, Brea, CA). Total cholesterol, white blood cell count (WBCc), low-density lipoprotein cholesterol (LDL-C), D-dimer, and serum creatinine were determined using standard quantitative assay techniques in the hospital clinical laboratory center according to the manufacturers' instructions.

\section{Statistical analysis}

Continuous variables were presented as mean \pm standard deviation (SD) or median and interquartile range, and categorical variables were displayed as counts or percentages. Student's $t$-test or Mann-Whitney test was used for the analysis of continuous variables and $\chi^{2}$-test for categorical variables. The correlation between cystatin $\mathrm{C}$ and hs-CRP was determined by the spearman rank correlation. Short- and long-term outcomes were determined using the Kaplan-Meier method and compared using the log-rank test. A logistic regression model was constructed for the multivariable analysis for markers, and cystatin C + hs-CRP (cystatin C + hs-CRP $=-8.40+0.62 *$ hs-CRP $+0.45^{*}$ cystatin C) was then generated. Receiver operating characteristic (ROC) analysis was used to compare the prognostic value of cystatin $\mathrm{C}$ and $\mathrm{D}$-dimer. SPSS 19.0 (SPSS Inc, Chicago, IL) and GraphPad Prism 6.5 (GraphPad Software Inc., CA) were used to perform statistical analysis. $P$ value $<0.05$ was considered statistically significant.

\section{CONFLICTS OF INTEREST}

The authors report that there are no conflicts of interest.

\section{REFERENCES}

1. Cifani N, Proietta M, Tritapepe L, Di Gioia C, Ferri L, Taurino M, Del Porto F. Stanford-A acute aortic dissection, inflammation, and metalloproteinases: a review. Ann Med. $2015 ; 47: 441-446$.

2. Nienaber CA, Clough RE, Sakalihasan N, Suzuki T, Gibbs R, Mussa F, Jenkins MT, Thompson MM, Evangelista A, Yeh JS, Cheshire N, Rosendahl U, Pepper J. Aortic dissection. Nat Rev Dis Primers. 2016; 2:16053.

3. Hagan PG, Nienaber CA, Isselbacher EM, Bruckman D, Karavite DJ, Russman PL, Evangelista A, Fattori R, Suzuki T, Oh JK, Moore AG, Malouf JF, Pape LA, et al. The International Registry of Acute Aortic Dissection (IRAD): new insights into an old disease. Jama. 2000; 283:897-903.

4. Fan XH, Huang B, Lu HS, Zhao ZH, Lu ZN, Yang YM, Zhang S, Hui RT. Impact of Admission White Blood Cell Count on Short- and Long-term Mortality in Patients With Type A Acute Aortic Dissection An Observational Study. Medicine. 2015; 94:7.

5. Vrsalovic M, Zeljkovic I, Presecki AV, Pintaric H, Kruslin B. C-reactive protein, not cardiac troponin $\mathrm{T}$, improves risk prediction in hypertensives with type A aortic dissection. Blood Pressure. 2015; 24:212-216. 
6. Tang N, Sun Z, Li D, Yang J, Yin S, Guan Q. Combined measurement of factor XIII and D-dimer is helpful for differential diagnosis in patients with suspected pulmonary embolism. Clin Chem Lab Med. 2017.

7. Li G, Wu XW, Lu WH, Cheng J, Wu XY, Ai R, Zhou ZH, Tang ZZ, Liao YH. High-sensitivity cardiac troponin T: A biomarker for the early risk stratification of type-A acute aortic dissection? Arch Cardiovasc Dis. 2016; 109:163-170.

8. Song Y, Xie Y, Liu F, Zhao C, Yu R, Ban S, Ye Q, Wen J, Wan H, Li X, Ma R, Meng Z. Expression of matrix metalloproteinase-12 in aortic dissection. BMC Cardiovasc Disord. 2013; 13:34.

9. Pitt B, Pfeffer MA, Assmann SF, Boineau R, Anand IS, Claggett B, Clausell N, Desai AS, Diaz R, Fleg JL, Gordeev I, Harty B, Heitner JF, et al. Spironolactone for heart failure with preserved ejection fraction. N Engl J Med. 2014; 370:1383-1392.

10. Kervella D, Lemoine S, Sens F, Dubourg L, Sebbag L, Guebre-Egziabher F, Bonnefoy E, Juillard L. Cystatin C Versus Creatinine for GFR Estimation in CKD Due to Heart Failure. Am J Kidney Dis. 2017; 69:321-323.

11. Liu J, Sukhova GK, Sun JS, Xu WH, Libby P, Shi GP. Lysosomal cysteine proteases in atherosclerosis. Arterioscler Thromb Vasc Biol. 2004; 24:1359-1366.

12. Shlipak MG, Sarnak MJ, Katz R, Fried LF, Seliger SL, Newman AB, Siscovick DS, Stehman-Breen C. Cystatin $\mathrm{C}$ and the risk of death and cardiovascular events among elderly persons. N Engl J Med. 2005; 352:2049-2060.

13. Lee M, Saver JL, Huang WH, Chow J, Chang KH, Ovbiagele B. Impact of elevated cystatin C level on cardiovascular disease risk in predominantly high cardiovascular risk populations: a meta-analysis. Circ Cardiovasc Qual Outcomes. 2010; 3:675-683.

14. Koenig W, Twardella D, Brenner H, Rothenbacher D. Plasma concentrations of cystatin $\mathrm{C}$ in patients with coronary heart disease and risk for secondary cardiovascular events: more than simply a marker of glomerular filtration rate. Clin Chem. 2005; 51:321-327.

15. Zhao L, Chai Y, Li Z. Clinical features and prognosis of patients with acute aortic dissection in China. J Int Med Res. 2017; 45:823-829.

16. Tang YF, Zhang GX, Liao ZL, Han L, Xu ZY. Surgical Treatment of Coronary Malperfusion with Acute Type A Aortic Dissection. Chin Med J (Engl). 2016; 129:1000-1002.

17. Elsayed RS, Cohen RG, Fleischman F, Bowdish ME. Acute Type A Aortic Dissection. Cardiol Clin. 2017; 35:331-345.

18. Coll E, Botey A, Alvarez L, Poch E, Quinto L, Saurina A, Vera M, Piera C, Darnell A. Serum cystatin C as a new marker for noninvasive estimation of glomerular filtration rate and as a marker for early renal impairment. Am J Kidney Dis. 2000; 36:29-34.

19. Fliser D, Ritz E. Serum cystatin C concentration as a marker of renal dysfunction in the elderly. Am J Kidney Dis. 2001; 37:79-83.
20. Gevorgyan MM, Voronina NP, Goncharova NV, Kozaruk TV, Russkikh GS, Bogdanova LA, Korolenko TA. Cystatin $\mathrm{C}$ as a Marker of Progressing Cardiovascular Events during Coronary Heart Disease. Bull Exp Biol Med. 2017; 162:421-424.

21. Hamzic-Mehmedbasic A. Inflammatory Cytokines as Risk Factors for Mortality After Acute Cardiac Events. Med Arch. 2016; 70:252-255.

22. Pascual-Figal DA, Caballero L, Sanchez-Mas J, Lax A. Prognostic markers for acute heart failure. Expert Opin Med Diagn. 2013; 7:379-392.

23. Taglieri N, Fernandez-Berges DJ, Koenig W, ConsuegraSanchez L, Fernandez JM, Robles NR, Sanchez PL, Beiras AC, Orbe PM, Kaski JC. Plasma cystatin C for prediction of 1-year cardiac events in Mediterranean patients with nonST elevation acute coronary syndrome. Atherosclerosis. 2010; 209:300-305.

24. Zhang J, Wu X, Gao P, Yan P. Correlations of serum cystatin $\mathrm{C}$ and glomerular filtration rate with vascular lesions and severity in acute coronary syndrome. BMC Cardiovasc Disord. 2017; 17:47.

25. Sukhova GK, Wang B, Libby P, Pan JH, Zhang Y, Grubb A, Fang K, Chapman HA, Shi GP. Cystatin C deficiency increases elastic lamina degradation and aortic dilatation in apolipoprotein E-null mice. Circ Res. 2005; 96:368-375.

26. Moulakakis KG, Sfyroeras GS, Papapetrou A, Antonopoulos CN, Mantas G, Kakisis J, Alepaki M, Mylonas $\mathrm{SN}$, Karakitsos P, Liapis CD. Inflammatory response and renal function following endovascular repair of the descending thoracic aorta. J Endovasc Ther. 2015; 22:201-206.

27. Curhan G. Cystatin C: a marker of renal function or something more? Clin Chem. 2005; 51:293-294.

28. Knight EL, Verhave JC, Spiegelman D, Hillege HL, de Zeeuw D, Curhan GC, de Jong PE. Factors influencing serum cystatin $\mathrm{C}$ levels other than renal function and the impact on renal function measurement. Kidney Int. 2004; 65:1416-1421.

29. Wen D, Zhou XL, Li JJ, Luo F, Zhang L, Gao LG, Wang LP, Song L, Sun K, Zou YB, Zhang CN, Hui RT. Plasma concentrations of interleukin-6, C-reactive protein, tumor necrosis factor-alpha and matrix metalloproteinase-9 in aortic dissection. Clin Chim Acta. 2012; 413:198-202.

30. Wen D, Du X, Dong JZ, Zhou XL, Ma CS. Value of D-dimer and $\mathrm{C}$ reactive protein in predicting inhospital death in acute aortic dissection. Heart. 2013; 99:1192-1197.

31. Wen D, Wu HY, Jiang XJ, Zhang HM, Zhou XL, Li JJ, Hui RT. Role of plasma C-reactive protein and white blood cell count in predicting in-hospital clinical events of acute type A aortic dissection. Chin Med J (Engl). 2011; 124:2678-2682.

32. Sakakura K, Kubo N, Ako J, Wada H, Fujiwara N, Funayama H, Ikeda N, Nakamura T, Sugawara Y, Yasu T, Kawakami M, Momomura S. Peak C-reactive protein level predicts long-term outcomes in type B acute aortic dissection. Hypertension. 2010; 55:422-429. 
33. Mori K, Tamune H, Tanaka H, Nakamura M. Admission Values of D-dimer and C-reactive Protein (CRP) Predict the Long-term Outcomes in Acute Aortic Dissection. Intern Med. 2016; 55:1837-1843.

34. Wang X, Ren HM, Hu CY, Que B, Ai H, Wang CM, Sun LZ, Nie SP. Predictors and in-hospital outcomes of preoperative acute kidney injury in patients with type A acute aortic dissection. J Geriatr Cardiol. 2016; 13:679-684.

35. Hiratzka LF, Bakris GL, Beckman JA, Bersin RM, Carr VF, Casey DE Jr, Eagle KA, Hermann LK, Isselbacher EM, Kazerooni EA, Kouchoukos NT, Lytle BW, Milewicz DM, et al. $2010 \mathrm{ACCF} / \mathrm{AHA} / \mathrm{AATS} / \mathrm{ACR} / \mathrm{ASA} / \mathrm{SCA} / \mathrm{SCAI} / \mathrm{SIR} /$ STS/SVM Guidelines for the diagnosis and management of patients with thoracic aortic disease. A Report of the American College of Cardiology Foundation/American
Heart Association Task Force on Practice Guidelines, American Association for Thoracic Surgery, American College of Radiology,American Stroke Association, Society of Cardiovascular Anesthesiologists, Society for Cardiovascular Angiography and Interventions, Society of Interventional Radiology, Society of Thoracic Surgeons, and Society for Vascular Medicine. J Am Coll Cardiol. 2010; 55:e27-e129.

36. Nienaber CA, Clough RE. Management of acute aortic dissection. Lancet. 2015; 385:800-811.

37. Yan Y, Zhou K, Wang L, Wang F, Chen X, Fan Q. Clinical significance of serum cathepsin $\mathrm{B}$ and cystatin $\mathrm{C}$ levels and their ratio in the prognosis of patients with esophageal cancer. Onco Targets Ther. 2017; 10:1947-1954. 